\title{
Broncoespasmo induzido por exercício
}

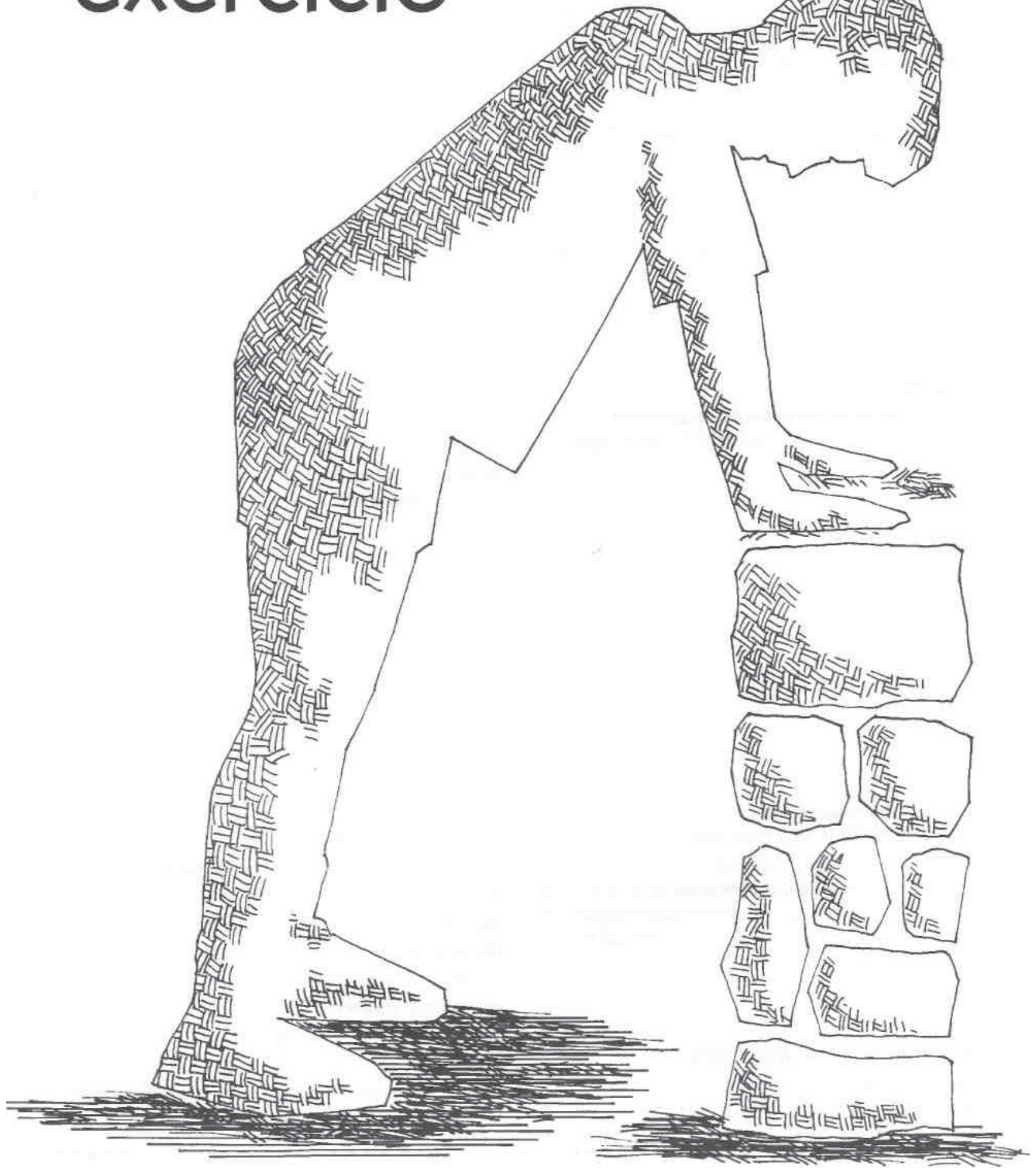


O broncoespasmo induzido por exercício (BIE), ou asma induzida por exercício (AIE), é uma obstrução das vias aéreas após vários minutos de exercício. Esta síndrome está presente em $90 \%$ dos indivíduos asmáticos, em 30 a 40\% dos alérgicos não asmáticos e em 3 a $10 \%$ dos atletas de várias modalidades, interferindo diretamente na prática esportiva destes. As hipóteses da perda de calor e água das vias respiratórias, da deficiência simpatoadrenal e da hiperemia reativa, as quais tentam explicar o mecanismo de desencadeamento do BIE, possuem pontos ainda não esclarecidos. $\mathrm{Na}$ relação asma/exercício, as particularidades tais como, tipo, intensidade, duração e forma de execução do exercício, são colocadas ora como provocadoras, ora como fatores preventivos do BIE, criando uma situação paradoxal a qual merece futuras pesquisas face às dúvidas que ainda permanecem nesta área. 


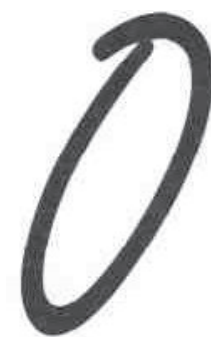

broncoespasmo induzido por exercício (BIE), ou asma induzida por exercício (AIE), é definido como uma obstrução das vias aéreas após vários minutos de exercício físico (Anderson, 1984). A caracterização do BIE se dá por uma redução igual ou superior a $10 \%$ do volume expiratório forçado, no primeiro segundo (VEFl), ou pico de fluxo expiratório (PEFR) após o exercício (Eggleston et al., 1979). O grau de BIE é expressado pela diferença percentual entre o valor em repouso e o valor mais baixo após o exercício, considerando-se o VEFl ou PEFR. Anderson (1986) classifica BIE, em termos de porcentagem de redução do VEFl ou PEFR, como média (10 a $25 \%$ ), moderada (25 a $35 \%$ ), moderada a severa (35 a $50 \%$ ) e severa (mais de 50\%).

O BIE pode ser identificado em $90 \%$ dos portadores de asma brônquica e em 35 a $40 \%$ dos indivíduos alérgicos nãoasmáticos (Kawabori et al., 1976) Entre os atletas sua prevalência é de 3 a $10 \%$ (Rice et al, 1985). Este fenômeno é um motivo freqüente para as crianças serem excluídas das aulas de educação física e práticas esportivas e representa a barreira mais comum para a prática de atividades físicas vigorosas, o que pode levá-los ao sedentarismo (Garfinkel et al., 1992). Por outro lado, pode-se observar a participação de atletas com BIE, asmáticos e não-asmáticos, nas competições de alto nível, em vários esportes (Voy, 1986).

O objetivo deste estudo é fazer um esclarecimento sobre o BIE, bem como fornecer subsídios para o trabalho de profissionais da área da educação física e esportes, tendo em vista que a relação asma/exercício não é bem entendida.

Para tal, abordar-se-á, no primeiro tópico, a fisiopatologia do BIE. No segundo tópico dar-se-á ênfase ao fator "exercício" quanto ao tipo, intensidade, duração e forma de execução do mesmo na relação de causa do BIE. No terceiro tópico o exercício será abordado como fator de auxílio ao asmático para o enfrentamento do BIE. Ao final serão feitas algumas considerações sobre o tema desenvolvido.

\section{A FISIOPATOLOGIA DO BIE}

Um episódio de BIE é diferente daqueles causados por outros fatores. Aumenta-se a resistência das vias aéreas. A condutância específica, o pico de fluxo respiratório (PEFR) e o volume expiratório forçado no primeiro segundo (VEFl), são reduzidos. Ocorre uma busca de ar pelo indivíduo, pois o volume residual e a capacidade residual funcional são regularmente aumentados. Ocasionalmente a capacidade pulmonar torna-se ampliada (Mefadden Jr., 1984).

Durante o exercício vigoroso, a demanda de Oxigênio (02) exigida aumenta a ventilação minuto (VE), fazendo com que predomine a respiração pela boca. A redução da função nasal, na respiração, prejudica o processo de condicionamento do ar inspirado. A ventilação aumentada, por sua vez, exacerba a perda de calor e água durante a respiração. Estas são as condições que terminam por desencadear o BIE em indivíduos suscetíveis (Bar-Or \& Inbar, 1992). Algumas hipóteses têm sido defendidas na tentativa de explicar o mecanismo de desencadeamento do BIE, a saber:

\section{Hipótese da perda}

de calor respiratório ou resfriamento

Esta hipótese enfatiza que o BIE tem como estímulo inicial a perda de calor respiratório, por convecção e evaporação 
durante o exercício, e hiperventilação. Esta perda, que ocorre para aquecer e umedecer o ar inspirado, provoca um resfriamento da mucosa bronquial com uma conseqüente estimulação das vias aéreas (Deal et al., 1979; Solway, 1985). O resfriamento pode ativar diretamente os receptores irritantes das vias aéreas e desencadear o broncoespasmo por reflexos vagais (Zeballos et al, 1978). A estimulação causa a liberação de agentes mediadores tais como: histamina, metacolina, fator quimiotáxico neutrofilico (NCF), leucotrienos sulfidopeptídicos e prostaglandinas contrateis das células pulmonares (Stick et al., 1990; Vanthenen et ai, 1991; Kikawa et al., 1992; Magnussen et al., 1992). Estes mediadores podem atuar de três formas, a saber: diretamente, contraindo a musculatura lisa dos brônquios; indiretamente, por reflexo neurogênico, causando o mesmo efeito; e pelo recrutamento de células inflamatórias ativadas, no local, para produzir as fases primária e tardia do BIE.

Deve-se salientar que a função dos mediadores na inflamação pode ser particularmente relevante em asmáticos que têm a obstrução do fluxo aéreo predominantemente nas vias aéreas periféricas. Entretanto, para os asmáticos que têm a obstrução do fluxo aéreo predominantemente nas vias aéreas centrais, os mediadores provocam o BIE através de mecanismos reflexos neurogênico, os quais ativam os receptores irritantes vagais (Strauss, 1978; Lee et al., 1984). Todavia é interessante observar que Finnerty et al. (1991) e Magnussen et al. (1992), não encontraram suporte para a teoria da atuação das prostaglandinas contrateis na gênese do BIE.

\section{Hipótese da perda}

de água respiratória ou ressecamento

Esta hipótese coloca que a perda de água da mucosa bronquial é a causa inicial do desencadeamento de mecanismo do BIE. Esta perda de água ocorre por evaporação e causa uma mudança na osmolaridade $\mathrm{e}$ permeabilidade do fluido que banha o trato respiratório, tornando-o hipo ou hiperos- molar. Isto resulta em um potente estímulo hipertônico para a broncoconstrição (Deal et al., 1979; Anderson et al., 1983; Hahn et al., 1984; Sheppard \& Eschenbacher, 1984; Bascom \& Bleecker, 1986). Esta estimulação também agirá na liberação de mediadores das células pulmonares, os quais irão induzir o broncoespasmo pelos mesmos processos descritos na hipótese anterior.

Entretanto, no que se refere a estas duas hipóteses, alguns estudos sugerem que a perda de calor respiratório apenas, ou a perda de água respiratória somente, são insuficientes para explicar a broncoconstrição induzida por exercício e hiperventilação, em condições ambientais normais, como sendo estímulos únicos (Strauss et al., 1978; Anderson, 1984; Eschenbacher \& Sheppard, 1985; Castel-Branco et al., 1991). A perda de calor e água respiratórios estão interligados em muitas circunstâncias. Podem ocorrer juntos, por exemplo, quando se respira ar muito frio, o que resulta no resfriamento da mucosa e mudança de osmolaridade do fluido superficial dos brônquios (Lee \& Anderson, 1985).

\section{Hipótese \\ da deficiência simpático-adrenal}

Tem sido sugerido que uma deficiência no aumento da concentração de catecolaminas plasmáticas, principalmente epinefrina (EP) plasmática, durante o exercício, pode ser um mecanismo que contribui para a estimulação do BIE (Stearns et ai, 1981), considerando-se a concentração de EP nos asmáticos como fator que pode conter a obstrução das vias aéreas.

Segundo Warren et al. (1982), nos indivíduos asmáticos que executam um teste de esforço suficiente para induzir broncoconstrição, há uma elevação moderada na NE e nenhuma elevação na EP. Entretanto, se os asmáticos executam um exercício mais vigoroso, observa-se uma elevação nas catecolaminas circulantes similar aos indivíduos normais (Larsson et al., 1982). Isto sugere que pode haver uma deficiência na
"Algumas hipóteses têm sido defendidas na tentativa de explicar 0 mecanismo de desencadeamento do BIE: 1- Perda de calor respiratório ou resfriamento; 2- Perda de água respiratória ou ressecamento; 3- Deficiência simpático* adremtl; 4- Hiperemia reativa". 
mobilização inicial de EP nos asmáticos. De acordo com Barnes (1986), a falha na elevação da EP plasmática, em resposta à broncoconstrição, poderia ser explicada através de uma menor secreção de EP pela medula adrenal ou através de uma maior remoção de EP pela circulação.

Neste sentido, o estudo de Knox et al. (1992) demonstra um aumento progressivo do VEF1 com o aumento da concentração de EP plasmática em asmáticos, sugerindo que a alteração da EP pode alterar a reatividade brônquica nestes indivíduos, e que a concentração de EP plasmática pode causar alguma proteção contra a broncoconstrição induzido pelo exercício em indivíduos normais e asmáticos. Por outro lado, Berkin et al. (1988), demonstraram que o aumento da EP e NE não diferiu significativamente entre asmáticos e não-asmáticos. Concluíram, então, que a reduzida resposta adrenal ao exercício não mostrou-se como um mecanismo importante na gênese do BIE.

\section{Hipótese \\ da hiperemia reativa}

Esta hipótese atribui o BIE a um mecanismo vascular, onde a limitação ou obstrução das vias aéreas é conseqüência de uma hiperemia reativa, a qual promove um edema na mucosa bronquial e, por conseqüência, a ativação da broncoconstrição (MacFadden, 1986, 1990).

Segundo Gilbert et al. (1988), a velocidade com que a temperatura das vias aéreas aumenta, após exercício, é maior nos asmáticos do que nos não-asmáticos. Apesar da queda de temperatura das vias aéreas ser similar entre estes indivíduos, para a mesma ventilação minuto (VE) e temperatura de ar inspirado, as vias aéreas dos asmáticos reaquecem duas vezes mais rápido no primeiro minuto de recuperação. Como a circulação bronquial é a principal fonte de calor das vias aéreas centrais e, desde que a hiperemia aumenta o calor local, pode-se inferir que a rede vascular tem uma função na etiologia do BIE (Mcfadden, 1990).

Outros dois fenômenos que são observados na relação asma/exercício, quando se fala dos mecanismos do BIE, merecem ser mencionados, porém não serão discutidos aqui. Trata-se do período refratário e da asma tardia.

O período refratário é o período no qual um exercício repetido, sob as mesmas condições, induz uma resposta asmática 50\% menor que a resposta ao exercício inicial. Assim, o indivíduo no qual as funções pulmonares retornarem a mais ou menos $15 \%$ do nível de pré exercício, será refratário ao BIE quando um segundo exercício for executado no intervalo de aproximadamente 2 horas (Edmunds et al., 1978).

A asma tardia é caracterizada por uma resposta asmática retardada, induzida pelo exercício, que ocorre de 3 a 9 horas após o exercício, podendo ser inibida por medicamentos administrados antes da execução do exercício (Bierman et al., 1984).

\section{O EXERCÍCIO COMO PROVOCADOR DO BIE}

A relação asma/exercício vem sendo estudada há vários anos, tendo em vista que o exercício pode ser visto, ora como provocador de asma, ora como fator de auxílio ao asmático na prevenção do BIE.

Considerando-se que os mecanismos patogênicos do BIE não são totalmente conhecidos, podendo ser variados e/ou múltiplos, o grau de broncoconstrição em resposta ao exercício poderá, também, variar de acordo com o tipo, a intensidade, a duração, a forma de execução e com o próprio organismo do executante (Strauss et al., 1977; Inbar et al., 1980; Bar-Yishay et al., 1982).

Em relação ao tipo de exercício, tem sido mencionado que a corrida livre causa mais obstrução bronquial do que a corrida numa esteira rolante, que o ciclismo 
(pedalar) revelou uma menor diminuição na função pulmonar do que a corrida, enquanto que a caminhada e a natação foram sempre menos efetivas em provocar BIE. Mencionase, também, que esportes como a natação e a canoagem, com uso predominante dos braços, são menos eficientes para induzir a obstrução bronquial (Fitch et al., 1976; Strauss et al., 1977; Inbar et ai, 1980; Bar-Yishay et ai, 1982). Quando a ventilação pulmonar, condições do ar inspirado e V02 são equivalentes, a corrida demonstrou ser mais asmagênica do que a natação (Bar-Yishay et al., 1982). Entretanto, quando as condições metabólicas (VE, V02 e percepção de esforço) são equivalentes, o ciclismo (pedalar) não mostrou-se mais asmagênico que a natação (Bundgaard et al., 1982). Porém, os mecanismos que justificam estas distinções não estão totalmente esclarecidos.

No que diz respeito à intensidade do exercício, considera-se que os fatores tais como, a carga de trabalho e a massa muscular em atividade, que são dependentes quando avaliadas pelo V02, determinam a taxa metabólica e conseqüentemente a resposta bronquial (Strauss et al., 1977). Isto poderia explicar a grande variabilidade do BIE entre pessoas de diferente sexos, idades, pesos, e em relação aos tipos de exercícios. Durante exercícios que duram de 6 a 8 minutos, com intensidade de 70 a $80 \%$ da capacidade aeróbica máxima (V02 máx.), bem como de 85 a $90 \%$ da freqüência cardíaca máxima (FCM), ocorrem os efeitos mais asmagênicos. Porém, a atividade de intensidade máxima não causa um alto grau de BIE. Uma explicação para isto pode ser que tal atividade não pode ser sustentada por muito tempo, ou por tempo bastante para ativar os mecanismos do BIE (Wilson \& Evans, 1981). Assim, as atividades com intensidade inferiores às mencionadas acima são indicadas para os asmáticos, pois possuem um risco menor de desencadear o BIE (Fitch \& Morton, 1988).

Alguns autores relacionam o grau de BIE, também, à duração do exercício. Por exemplo, um exercício com duração de 6 a
8 minutos induziu um grau de broncoconstrição maior do que um protocolo mais curto ou mais longo em uma intensidade que equivalia de 60 a $80 \%$ da capacidade aeróbica máxima (Wilson \& Evans, 1981). Entretanto, não é sabido se esta duração é adequada para o exercício em outras intensidades, pois Inbar et al. (1980), mostraram que asmáticos adultos jovens tiveram uma queda no fluxo expiratório médio, após uma corrida supramáxima na esteira rolante, chegando à exaustão aos 50 segundos, maior, portanto, do que a exaustão obtida numa corrida de 7 minutos, numa intensidade submáxima.

Quanto à forma de execução do exercício, indica-se que a forma intervalada causa menos BIE do que a forma contínua (Svenonius et ai, 1983), este autor indica a combinação de 2 minutos de esforço intercalados com 2 minutos de repouso. Esta indicação orienta-se no fato de que a repetição seriada do exercício provoca uma broncoconstrição menor nos exercícios seguintes, segundo as teorias do período refratário (Edmunds et al., 1978; Sterns, 1981).

\section{OEXERCÍCIO COMOPREVENTINODOBE}

A resposta à questão de que o exercício pode prevenir uma crise de BIE não está definida, pois os resultados das pesquisas nesta área deixam alguns pontos sem esclarecimento. Com a prática de atividades físicas pode-se melhorar a tolerância ao exercício e, conseqüentemente, os ganhos em termos de capacidade aeróbica podem aumentar o limiar de desencadeamento do BIE (Svenonius et al., 1983; Haas et al., 1987). Isto permite que o indivíduo possa praticar certas atividades físicas, evitando o BIE. Porém existem dúvidas de que os benefícios do treino físico possam diminuir ou prevenir o BIE (McFadden Jr., 1984; Fitch et ai, 1986 Cochrane \& Clarck, 1990).

Neste sentido, Fitch \& Morton (1988) relatam que os exercícios aeróbicos, praticados de forma freqüente e regular a uma intensidade moderada e submáxima, produ-

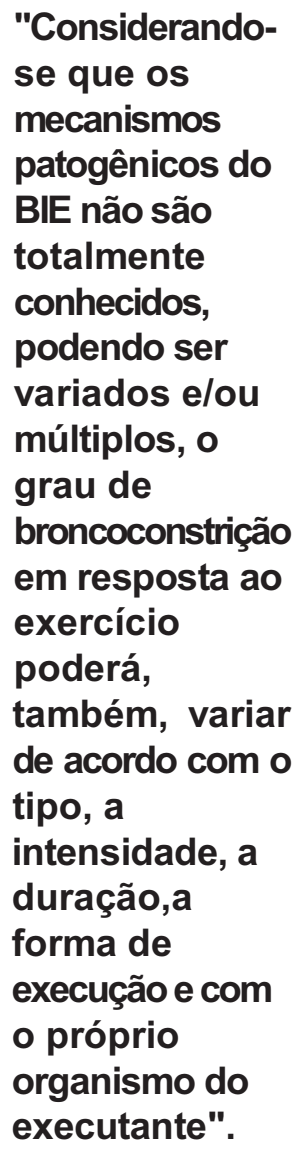


zem benefícios fisiológicos aos asmáticos. Assim, com a aptidão aeróbica aumentada, níveis mais altos de intensidade são exigidos para provocar BIE.

Entretanto, Nickerson (1989) reporta que, em seu estudo realizado em 1983 com crianças asmáticas, observou-se uma melhora na aptidão física que as tornaram aptas a participar de várias atividades físicas. Mas que nenhuma mudança ocorreu em seus sintomas diários, pico de fluxo, funções pulmonares em repouso e propensão a desenvolver BIE.

Os atletas asmáticos têm obtido resultados surpreendentes em esportes que exigem esforços ventilatórios altos. Para exemplificar, Haas et al. (1985) montaram um experimento para determinar se existe um mecanismo induzido pelo exercício nos indivíduos asmáticos que os compensem de suas limitações de fluxo aéreo, e se tal mecanismo pode ser potencializado pelo grau de aptidão física destes indivíduos. Os resultados mostraram um fluxo aéreo expiratório aumentado nas funções pulmonares dos asmáticos durante o exercício, devido à condutância aumentada das vias aéreas, e que os asmáticos treinados mantiveram este fluxo aumentado do início ao fim do exercício, enquanto que os não treinados falharam neste processo. Isto sugere que os mecanismos responsáveis pelo BIE são inici-

"Embora o número de pesquisas seja grande, $o$ gatilho pelo qual o mecanismo ou mecanismos do BIE são ativados não estão totalmente esclarecidos, e as tentativas de explicação são, às vezes controversas". ados durante o exercício, com o efeito extensivo do exercício no fluxo aéreo, refletindo um balanço entre a broncodilatação e a broncoconstrição induzidas pelo exercício. Estes autores afirmam que a capacidade de manter maiores reservas de fluxo e, conseqüentemente, altos níveis de ventilação, acoplado à redução no trabalho respiratório durante o exercício, resultando em broncodilatação, pode ser suficiente para explicar porque alguns indivíduos asmáticos são hábeis para competir com sucesso nos esportes, apesar de sua função pulmonar de préexercício ser significativamente diminuída.

Em outro estudo de Haas et al. (1987), um programa de treino aeróbico para asmáticos, com duração de 12 semanas, causou os seguintes efeitos:

1) Aumento da broncodilatação induzida pelo exercício. Este aumento foi justificado, pelo autor, como sendo uma conseqüência do aumento direto da sensitividade do adrenorreceptor beta 2, causado pelas catecolaminas, e do aumento da estimulação do adrenorreceptor beta 2 , causado pelas catecolaminas, e do aumento do número de receptores brônquicos.

2) Aumento do limiar de broncoconstrição induzida pelo exercício, isto é, exigência de uma quantidade maior de esforço físico para induzir o broncoespasmo.

3) Aumento da ventilação voluntária máxima (WM), ou seja, maior capacidade de ventilação máxima por unidade de tempo.

4) Melhora na relação freqüência cardíaca (FC)/carga de trabalho (CT), isto é, exigência de uma menor FC para uma determinada $\mathrm{CT}$.

5) Aparente diminuição da sensitividade das vias aéreas, sem alterar a reatividade destas.

Entretanto, quanto à reatividade bronquial, pode-se perceber alguns achados controversos, senão vejamos. Ao contrário dos resultados de Knox et al. (1992), os quais sugerem que a modificação do EP plasmática pode alterar a reatividade brônquica nos asmáticos, para Cochrne \& Clark (1990), apesar do treino físico submáximo com intensidade controlada produzir uma melhora significativa na aptidão cardiorespiratória nos asmáticos, a reatividade bronquial não específica permanece sem alteração. As melhoras no BIE, após o treino, são mais devido à redução da ventilação minuto, a qual resulta em um decréscimo no estímulo para o BIE, do que uma alteração no processo fisiopatológico.

\section{CONSDERACÕESFNAS}

Embora o número de pesquisas seja grande, o gatilho pelo qual o mecanismo ou mecanismos do BIE são ativados não estão totalmente esclarecidos, e as tentativas 
de explicação são, às vezes, controversas. Necessita-se, porém, que mais estudos sejam feitos para que este fenômeno seja melhor conhecido. Entretanto, há de se reconhecer as dificuldades de pesquisá-lo "in vivo".

Outro fator importante a considerar é que a falta de conhecimento da relação asma/exercício pode servir como obstáculo para que os asmáticos participem da prática esportiva, percebendo uma aptidão física deficiente que pode levá-los ao sedentarismo. Considerando-se o fato destes indivíduos possuírem aptidão física ou capacidade para exercitarem-se o suficiente para participarem de programas de exercícios especiais, pode-se acreditar na possibilidade dos efeitos benéficos destes programas.

Deve-se considerar, também, que o potencial para se alcançar níveis de aptidão física equivalentes entre asmáticos e não asmáticos é controverso, e os fatores que determinam as habilidades dos asmáticos para o exercício não são bem conhecidos (Garfinkel et al., 1992). Portanto, torna-se evidente a necessidade do conhecimento do tema para que a participação dos asmáticos nas atividades físico-esportivas de sua preferência seja benéfica.

\section{RER̂́NDASBBOCRÁACAS}

ANDERSON, S.D. et alli. Exercise-induced asthma (EIA) without airway cooling? American Review of Respiratory Disease. v.127 228, 1983.

ANDERSON, S.D. Is there a unifying hypothesis for exercise-induced asthma? Journal of Allergy and Clinical Immunology. San Francisco, v. 73: 660-665, 1984.

. EIB: new thinking and current management. Journal of Allergy and Clinical Immunology. v.77: 40-61, 1986.

BARNES, P.J. et alli. Endogenous catecholamines and asthma. Journal of Allergy and Clinical Immunology. V.77: 791-795, 1986.

BAR-OR, O \& INBAR, O. Swimming and asthma: Benefits and deleterious effects Sports Medicine, v. 14, n. 6: 397-405, 1992.
BAR-YISHAY, E. et alli. Differences between swimmers and running as stimuli for exercise-induced asthma. European Journal of Applied Phisiology an Occupational Phisiology, Berlim, 48, n. 3: 387-397, 1982.

BASCOM, R. \& BLEECKER, E.R.. Bronchoconstriction induced by destiled water. American Review of Respiratory Disease, New York, v. 134: 248-253,1986.

BERKIN, K.E. et alli. Circulating adrenaline and noroadrenalina concentrations during exercise in patients with exercise induced asthma and normal subjects. Thorax. London, v. 43, n. 4: 295-299, 1988.

BIERMAN, W.C. et alli. Caracterization of the late response in exercise induced asthma. Journal of Allergy and Clinical Immunology. San Francisco, v. 74, n. 5: 701-706, 1984.

BUNDGAARD et alii. Exercise-induced asthma after swimming and bicycle exercise. European Journal of Respiratory Disease. v. 63: 245-248, 1982.

CASTEL-BRANCO, M.G. et alii. Bronchial hiperreactivity in young asthmatic ? comparison between exercise and ultrasonically nebulized nonisotic Solutions. Journal of Allergy and Clinical Immunology. v. 87, n. 1: 337, 1991.

COCHRANE, L.M. \& CLARCK, C.J.. Benefits and problems of a training programme for asthmatic patients. Thorax. London, v. 45, n. 5: 345-351, 1990.

DEAL Jr., E.C. et alii. Role of repiratory heat exchange in production of exercise-induced asthma. Journal of Applied Physiology. Bethesda, v. 46, n. 3: 467- 475,1979.

EDMUNDS, A.T. et alii. The refractory period after exercise-induced asthma: Its duration and relation to the severity of exercise. American Review of Respirary Disease. New York, v. 117: 247-255, 1978.

EGGLESTON, P.A. et alii. Guidelines for the methodology of exercise challenge testing of asthmatics. Journal of Allergy and Clinical Immunology. v. 64: 642-645, 1979.

FINNERTY, J.P. et alii. Effect of GR32191, a potent thromboxane receptor agonist on exercise induced bronchoconstriction in asthma. Thorax. v. 46, n. 3: 190-192, 1991.

FITCH, K.D. et alii. Effects of swimming training on children with asthma. Archives of Disease in childhood. v. 51: 190-195, 1976.

FITCH, K.D.. The use of anti-asthmatic drugs. Do they affect sports performance? Sports Medicine, v. 3: 136$150,1986$. 
FITCH, K.G. \& MORTON, R.. Resspiratory disease. In: DIRIX, A. et alii. (ed.) The Olimpic Book of Sports Medicine. London, Blackwell Scientific Publications, 1988.

GARFINKEL, S.K. et alii. Physiologic and nonphysiologic determinants of aerobic fitness in mild to moderate asthma. American review of Respiratory Disease. New York, v. 145: 741-745, 1992.

GILBERT, LA. et alii. Intra-airway thermodynamics during exercise and hiperventilation in asthmatics. Journal of Applied Physiology. v. 54: 2167-2174, 1988.

HAAS, F. et alii. Effects of physical fitness on expiratory airflow in exercising asthmatics people. Medicie and Sciences in Sports and Exercise. Indianopolis, v. 17, n.5: 585-592, 1985.

Effect of aerobic training on for-

ced expriratory airflow in exercising asthmatic humans. Journal of Applied Physiology. Bethesda, v.63, n. 3: 1230-1235, 1987.

HAHN, A. et alii. A reinterpretation of the effect of temperature and water content of the inspired air in exercise-induced asthma. American review of Respiratory Disease. New York, v. 130: 575-579, 1984.

INBAR, O. et alii. Breathing dry or humid air and exercise-induced asthma during swimming. European Journal of Applied Physiology. Berlim, v. 44: 43-50, 1980.

KAWABORI, I. et alii. Incidence of exercise-induced asthma in children. The Journal of Allergy and Clinical Immunology. v. 58: 447, 1976.

KIKAWA, Y. et alii. Urinary leukotriene E4 after exercise challenge in children with asthma. Journal of Allergy and Clinical Immunology. San Francisco, v. 89, n. 6: 1111-1119, 1992.

KNOX, A.J. et alii. Modification of bronchial reactiwity by physiological concentrations of plasma epinephrine. Journal of Allergy and Clinical Immunology. Bethesda, v. 73, n. 3: 1004-1007, 1992.

LARSSON, K. et alii. Symphatoadrenal reactivity in exercise-induced asthma. Chest. v. 82: 561, 1982.

LEE, T.H. et alii. Mediators in exercise-induced asthma Journal of Allergy and Clinical Immunology. San Francisco, v. 73, n. 5: 634-639, 1984.

LEE, T.H. \& ANDERSON, S.D.. Heterogeneity of mechanisms in exercise-induced asthma. Thorax. v. 40, n. 7: 481-487, 1985.

MAGNUSSEN, H. et alii. Effects of a thromboxane- receptor antagonist, BAY u 3405, on prostaglandin D2 and exercise-induced bronchoconstriction. Journal of Allergy and Clinical Immunology. San Francisco, v. 89, n. 6: 1119-1126, 1992.

McFADDEN, Jr., E. R.. Exercise performance in the asthmatic. American Review of Respiratory Disease. New York, n. 129, suppl: 84-87, 1984.

McFADDEN Jr., E.R. et alii. Post exertional airway rewarming and thermally induced asthma. Journal of Clinical Investigation. v. 78: 18-25, 1986.

McFADDEN, E.R. Exercise-induced asthma as a vascular phenomenon. lancet. London, v. 335:880-883,1990.

NICKERSON, B.G. Asthmatic patients and those with exercise-induced bronchoespasm In: FRANKLIN, BA. et alii. (ed.) Exercise in modern medicine. Baltimore, Willians \& Wilkins, cap. 10: 192-203, 1989.

RICE, S.G. et alii. Identification of exercise-induced asthma among intercollegiate athletes. Annals of Allergy. v. $55: 790-793,1985$

SHEPPARD, D. \& ESCHENBACHER, W.L. Respiratory water loss as a stimulus to exercise-induced broncoconstriction. Journal of Allergy and Clinical Immunology. San Francisco, v. 73, n. 3: 640-642,1984.

SOLWAY, J et alii. Breating pattern affects airway wall temperature during cold air hiperpnea in humans. American Review of Respiratory Disease. New York, v. 132: 853-857, 1985

STERARNS, D.R. et alii. Reanalysis of refractory period in exertional asthma. Journal of Applied Physiology. Bethesda, v. 50, n. 3: 503-508, 1981.

STICK, S.M. et alii. Bronchial responsiveness to histamine ininfantes and older childrens. American Review of Respiratory Disease. New York, v. 142:1143-1146,1990.

STRAUSS, R.H. et alii. Comparison of arm versus leg work in induction of acute episodes of asthma. Journal of Applied Physiology. Bthesda, v. 42, n. 4: 565-570, 1977.

. Influence of heat and humidity on the airway obstruction induced by exercise in asthma. The Journal of Clinical Investigation. New York, v. 61: 433-440, 1978

SVENONIUS, E. et alii. Improvement after training of children with exercise-induced asthma. Acta Paediatica Scandinavica. v. 72: 23-30, 1983.

VANTHENEN, et alii. Effect of inhaled budesonide on bronchial reactivity to histamine, exercise and eucapnic dry air hiperventilation in patiertts with asthma. Thorax London, v. 46, n. 11: 811-816, 1991. 
VIRANT, F.S.. Exercise-induced bronchospasm: epidemiology, pathopysiology, and therapy. Medicine Science in Sports and Exercise. v. 24, n. 8: 851855,1992 .

VOY, R.O.. Olympic committe experience with exercise-induced bronchospasm. Medicine Science in Sports and Exercise. 328-330, 1986.

ZEBALLOS, RJ., et alii. The role of hyperventilation in exercise-induced bronchoconstriction. American Review of Respiratory Disease. v. 118: 877-884, 1983.

WARREN, J.B. et alii. Blunted symphatoadrenal response to exercise in asthmatics subjects. Britsh Journal of Disease Chest. v. 76: 147, 1982.

WILSON, B.A. \& EVANS, J.N.. Standardization of work intensity for evaluation of exercise-induced bronchoconstriction. European Journal of Applied Phbisiology. Berlim, v. 47: 289-294, 1981.

\section{UNITERMOS}

Exercício - Asma - Broncoespasmo

Prof. Antônio José Natali

Prof. Mestre da Universidade de Viçosa

Prof. Dilson José E. Rassier

Mestre em Educação Física

Prof. Eduardo Henrique De Rose

Prof. Doutor da ESEF-UFRGS 